galaxies took to form. The big bang picture would be in trouble if the expansion time was less than the age of our Galaxy. Second, discrepancies which are such a large multiple of the quoted errors do not enhance the status of observational astronomy. At the moment the onus seems to be on the opponents of the Sandage and Tammann scale to assemble a similar body of observational data. Theoreticians will have to maintain the scepticism about $H_{0}$ that the wiser among them have always shown in the past.

\section{RNA and generation of positional information}

\section{from Peter Lawrence}

Most biochemical investigation on developing systems is just conventional biochemistry on a system which happens to be developing, and is not relevant to the understanding of development in terms of the controlling molecules involved. (After all, even developing animals have to make proteins, send acids scooting round the Krebs cycle and so on). Conversely, specifically developmental phenomena, such as regulation are difficult to approach from a biochemical point of view. But several insects, including the tiny dipteran midge Smittia, undergo what Sander (Wilhelm Roux Archiv., 167,$336 ; 1971$ ) has called "negative regulation". An embryo regulates by reacting to experimental damage, such as removal of tissue, by respecification of cells so that normal development is restored. In negative regulation, however, respecification proceeds abnormally so that a monster develops. These striking changes in Smittia have provided investigators with a handle on the molecular mechanisms which may be involved in specifying that pattern.

Normally the insect consists of a number of segments arranged in order from head to tail; each segment is uniquely patterned. The segment order in most insect eggs becomes defined at blastoderm (when the embryo is just a hollow ball of cells) and depends only on the positions of the cells forming at the surface of the egg, not on the lineage of their nuclei. There is thus positional information in the egg. Experiments on other insects (see Sander, Ciba Foundation Symp., 29, 241 ; 1975) suggest that the positional information is in the form of a gradient which is made by an interaction between two determinants, an anterior and a posterior one, at the poles of the egg. Kalthoff working in Sander's laboratory (Kalthoff, Roux. Arch., $168,63 ; 1971)$ discovered that local ultraviolet irradiation of the anterior pole of cleaving Smittia eggs (the nuclei were untouched by irradiation) caused up to $100 \%$ of the embryos to change their segment pattern from 123456789 to 98766789 (Fig. 1). This aberrant pattern is called a 'double abdomen'. The targets to ultraviolet were localised in the egg cytoplasm and distributed unevenly in the anterior third of the egg, becoming most concentrated at the anterior tip. The damage done by ultraviolet was not violent: nuclei subsequently entered the irradiated region and developed normally into cells. Only the positional information, and hence the segment pattern, seemed to be altered. Kalthoff found the ultraviolet damage could be repaired by visible light so that normal embryonic development was restored; this suggested that nucleic acid might be the target. Action spectra (Kalthoff, Photochem. Photobiol., 18, 355; 1973) showed peaks in both the protein and the nucleic acid regions.

This line has now been taken one step further by Kandler-Singer and Kalthoff (Proc. natn. Acad. Sci., 73, $3739 ; 1976)$. They were able to produce double abdomens by gently puncturing the anterior pole of the egg in water containing RNase $\left(0.8 \mu \mathrm{g} \mathrm{ml}^{-1}\right)$. Although about half the embryos failed to develop at all, $47 \%$ of those which did made double abdomens. In water only some $5 \%$ failed to develop and none grew up with double abdomens. With an elegant control they demonstrated that it was actually the RNase activity which was responsible for altering the segment pattern. They treated the eggs with two fragments of RNase each of which is inactive separately (RNase $\mathrm{S}$ (active) is split by TCA fractionation into S-peptide (inactive) and S-protein (hardly active)). When solutions of the two fragments are mixed RNase activity returns. The percentage of double abdomens was nil with S-peptide, 1 with the $\mathrm{S}$ protein

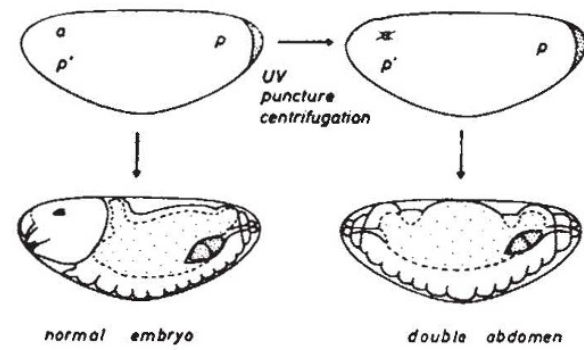

Diagrammatic representation of the induction of a double abdomen in Smittia eggs by ultraviolet irradiation of the anterior pole region, puncture at the anterior pole or centrifugation with the long egg axis parallel to the centrifugal force. (From Insect De velopment, edited by Lawrence, P. Blackwells Scientific, Oxford and London, 1976.) and about 40 both with complete RNase $S$ and with the two fragments dissolved together. Puncturing of the eggs elsewhere than at the anterior pole produced no double abdomens. Double abdomens were formed only when the experiment was performed up to the blastoderm stage of embryogenesis. These results together with those mentioned earlier, strongly suggest that an RNA moiety is an essential component in the generation of positional information in the egg. Kandler-Singer and Kalthoff suggest it might be mRNA that is translated during cleavage.

The next stage in this investigation will ideally be the development of a positive assay in which the damage done by ultraviolet will be repaired by the injection of extracts into the anterior pole of the egg. This could lead to the first isolation of a molecule specifically involved in the generation of positional information and perhaps to some inkling as to what gradients are in molecular terms.

\section{Structural gene for sex pilin}

from J. P. Beard

A RECENT paper from Charles Brinton's laboratory in Pittsburgh reports on an important aspect of the mechanism whereby 'male' Gram-negative bacteria are able to transfer genetic material to 'female' bacteria by conjugation. This subject has been of great interest to microbiologists for many years, since not only does the conjugation process provide a useful research tool for probing the genetic composition of Escherichia coli and related species, but it is also usually mediated by genes carried on plasmids in the male or donor cell. Plasmids are small extrachromosomal pieces of DNA, carried by a wide variety of bacterial genera, which are capable of autonomous replication in the host cell, and which can often transfer both themselves and chromosomal DNA in the conjugation process. Plasmids are also important as vectors of multiple antibiotic resistance genes, or of genes which confer other important properties on the host such as colicinogeny or enterotoxin production (see, for example, the Ciba Foundation Symposium on Bacterial Episomes and Plasmids, J. and A. Churchill, 1969), and have recently taken on a new lease of life as vectors for "genetic engineering", currently the subject of intense public discussion.

Studies of one such plasmid, the sex factor F (Hayes, The Genetics of Bacteria and their Viruses, Blackwell, 